\title{
SEA OIL SPILL DETECTION USING SELF-SIMILARITY PARAMETER OF POLARIMETRIC SAR DATA
}

\author{
Shengwu Tong, Qihao Chen, Xiuguo Liu* \\ Faculty of Information Engineering, China University of Geosciences (Wuhan), Wuhan 430074, China \\ cug_tsw@163.com(S.T.); chenqihao@cug.edu.cn; liuxg318@hotmail.com(X.L.)
}

\author{
Commission III, WG III/1
}

KEY WORDS: Oil Spill Detection, PolSAR Image, Self-Similarity Parameter, Polarimetric Features, Look-alikes, Remote Sensing

\begin{abstract}
:
The ocean oil spills cause serious damage to the marine ecosystem. Polarimetric Synthetic Aperture Radar (SAR) is an important mean for oil spill detections on sea surface. The major challenge is how to distinguish oil slicks from look-alikes effectively. In this paper, a new parameter called self-similarity parameter, which is sensitive to the scattering mechanism of oil slicks, is introduced to identify oil slicks and reduce false alarm caused by look-alikes. Self-similarity parameter is small in oil slicks region and it is large in sea region or look-alikes region. So, this parameter can be used to detect oil slicks from look-alikes and water. In addition, evaluations and comparisons were conducted with one Radarsat-2 image and two SIR-C images. The experimental results demonstrate the effectiveness of the self-similarity parameter for oil spill detection.
\end{abstract}

\section{INTRODUCTION}

With the development of marine transport and offshore oil industry, oil spill events happened more and more frequently, which caused by pipeline rupture, offshore oil tanker, and drilling platform leakage. Marine oil spill pollution not only causes serious damage to the marine ecological environment, but also brings huge losses to the local economy. Therefore, it is necessary to monitor marine oil spill, and oil spill detection is of great help for the pollution treatment. With the continuous development of technology, remote sensing technology has gradually become one of the most important and effective means of oil spill detection. Among the many remote sensing sensors, SAR has better detection performance because of its strong penetration, active imaging, all-weather and all-time capability (Brekke et al., 2005, Li et al., 2017).

On the ocean surface, oil slicks can damp the capillary waves and short gravity waves, which reduce the roughness and dielectric constant of the ocean surface. Therefore, oil spill region will from dark area in SAR image, by identifying the dark areas in SAR image can detect oil spill. However, there are other natural phenomena which cause dark areas in SAR images (Migliaccio et al., 2005), including natural biological slicks, low-wind areas, rain clouds, etc. These called look-alikes phenomena also form the dark spot area in the SAR image like oil slicks, which increase the difficulty of SAR oil spill detection (Migliaccio et al., 2007, Migliaccio et al., 2012).

Polarimetric SAR extract the scattering behavior of observed target by combine information in the different channels. The polarimetric information is propitious to distinguish between oil slicks and look-alikes (Migliaccio et al., 2012). There are different physicochemical properties and scattering behavior between oil slicks and look-alikes. In clean ocean and look-alikes, the backscattering mechanism is mainly Bragg scattering, which is influenced by capillary waves and short gravity waves. In oil slicks, the capillary waves and short gravity waves are restrained because of the stickiness of oil slicks. So, the backscattering of oil slicks is mainly non-Bragg scattering, and it is more complex than clean ocean and look-alikes (Nunziata et al., 2011, Migliaccio et al., 2012). Based on this theory, there are many studies of oil spill detection based on polarimetric features in recent years, which detect oil slicks by the differences of polarimetric scattering mechanism between oil slicks and non-oil slicks (such as look-alikes and water) (Migliaccio et al., 2007, Wang et al., 2010, Nunziata et al., 2011, Zhang et al., 2011, Liu et al., 2011, Nunziata et al., 2013, Skrunes et al., 2014, Migliaccio et al., 2015, etc.).

In order to effectively improve the accuracy of the oil spill detection and reduce the false alarm caused by look-alikes, we introduced the self-similarity parameter to detect oil spills. Selfsimilarity parameter is a special case of similarity, which can describe the randomness of the target scattering mechanism ( $\mathrm{Li}$ et al., 2015). On the ocean surface, the randomness of oil slicks is higher than water and look-alikes because of its complex nonBragg scattering mechanism (Nunziata et al., 2011, Migliaccio et al., 2012). Self-similarity parameter can reflect this difference. Therefore, self-similarity parameter is used to detect oil spills and reduces the false alarm caused by look-alikes in this paper.

\section{METHODOLOGY}

The similarity parameter was proposed to measure the similarity between tow scattering matrices by Yang (Yang et al., 2001), which extract the scattering mechanism of targets by calculating the similarity between targets and the particular target (sphere, diplane reflector, left/right helix, etc.) (Yang et al., 2001, Chen et al., 2010). It has achieved good result that utilized similarity parameter to detect ground target and ship target (Yang et al., 2004). The self-similarity parameter $\left(r r r_{s}\right)$ is a new parameter defined based on similarity parameter by Li Dong (Li et al., 2015), which is related to its own scattering matrix and not associated with the particular target.

In polarimetric $\mathrm{SAR}$, the scattering matrix $S$ is defined as:

$$
\boldsymbol{S}=\left[\begin{array}{ll}
S_{H H} & S_{H V} \\
S_{V H} & S_{V V}
\end{array}\right]
$$


In single-look data, the similarity parameter $r$ of two matrixes $S$ is defined as (Yang et al., 2001):

$$
r\left(\boldsymbol{S}_{1}, \boldsymbol{S}_{2}\right)=\frac{\left|\boldsymbol{k}_{1}^{H} * \boldsymbol{k}_{2}\right|^{2}}{\left\|\boldsymbol{k}_{1}\right\|^{2} *\left\|\boldsymbol{k}_{2}\right\|^{2}}
$$

where $H$ is conjugate transpose, $\boldsymbol{k}$ is the target vector of the scattering matrix $\boldsymbol{S}$ and defined as:

$$
\boldsymbol{k}=\frac{1}{\sqrt{2}}\left[S_{H H}+S_{V V}, S_{H H}-S_{V V}, 2 S_{H V}\right]^{t}
$$

Where $t$ is transpose. In multi-look data, the similarity parameter $r r r$ of a mixed scatterer $\boldsymbol{T}$ and a canonical mixed scatterer. $\boldsymbol{T}_{c}$ is defined as (Li et al., 2015):

$$
\operatorname{rrr}\left(\boldsymbol{T}, \boldsymbol{T}_{c}\right)=\frac{\operatorname{tr}\left(\boldsymbol{T} \boldsymbol{T}_{c}^{H}\right)}{\operatorname{tr}(\boldsymbol{T}) \operatorname{tr}\left(\boldsymbol{T}_{c}\right)}
$$

where $\operatorname{tr}(\cdot)$ is the trace of matrix. If $\boldsymbol{T}$ and $\boldsymbol{T}_{c}$ are two rank-1 mixed scatterer, $\boldsymbol{T}=\boldsymbol{k} \cdot \boldsymbol{k}^{H}$ and $\boldsymbol{T}_{\boldsymbol{c}}=\boldsymbol{k}_{\boldsymbol{c}} \cdot \boldsymbol{k}_{\boldsymbol{c}}^{H}$, then Eq. (4) can be further written as (Li et al., 2015):

$$
\left(\boldsymbol{T}, \boldsymbol{T}_{c}\right)=\frac{\left|\boldsymbol{k}^{H} * \boldsymbol{k}_{c}\right|^{2}}{\|\boldsymbol{k}\|^{2} *\left\|\boldsymbol{k}_{c}\right\|^{2}}=r\left(\boldsymbol{S}, \boldsymbol{S}_{c}\right)
$$

In this case, $r r r$ and $r$ are equivalent. The self-similarity parameter can be identified by similarity parameter $r r r$, the selfsimilarity parameter $r r r_{S}$ of matrix $\boldsymbol{T}$ is defined as (Li et al., 2015):

$$
r r r_{s}(\boldsymbol{T})=r r r(\boldsymbol{T}, c * \boldsymbol{T})=\frac{\sum_{i=1}^{3} \lambda_{i}^{2}}{\left(\sum_{i=1}^{3} \lambda_{i}\right)^{2}}
$$

where $c$ is an arbitrary number, $\lambda_{1}, \lambda_{2}$, and $\lambda_{3}$ denotes the first eigenvalue, the second eigenvalue, and the third eigenvalue of $\boldsymbol{T}$, respectively.

When the target is a single scatterer, matrix $\boldsymbol{T}$ has only one nozero eigenvalue, then $r r r_{s}$ reaches the maximum 1 . When the target is a fully randomly noisy scatterer, matrix $\boldsymbol{T}$ has three equal eigenvalues, then $r r r_{s}$ reaches the minimum 1/3. For the general mixed scatterer, $r r r_{s}$ resides between $1 / 3$ and 1 .

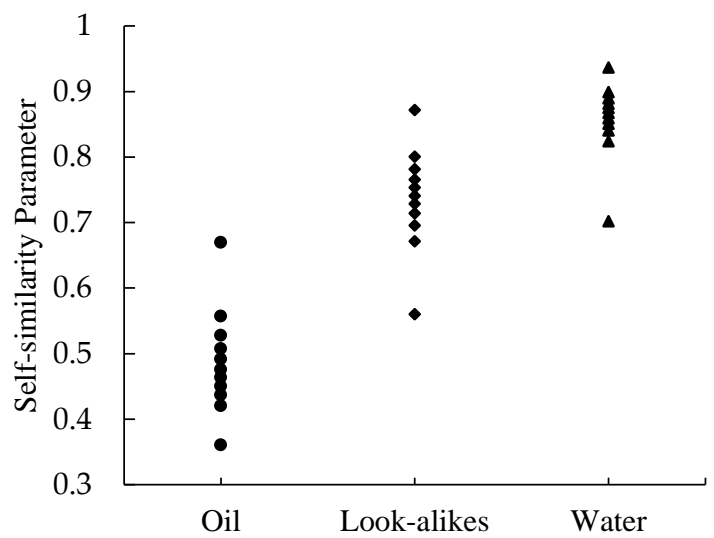

Figure. 1. The value distribution of $r r r_{s}$ in the oil slicks, lookalikes, and water.
In the clean water, due to the influence of capillary waves and short gravity waves, the scattering mechanisms are mainly Bragg scattering with low depolarization and low random (Van Zyl, 1989, Nunziata et al., 2011), $\lambda_{1}$ is much large than $\lambda_{2}$ and $\lambda_{3}$, so the value of $r r r_{s}$ is higher. In the look-alikes, the thickness of the slicks is thin, so the damping effect on the Bragg scattering is weak, and it is still in the scatter state of low depolarization and low random (Skrunes et al., 2014), so the value of the $\mathrm{rrr}_{s}$ is still high. In the oil slicks, the damping effect of the oil slicks is very strong, which damps the Bragg scattering leading to a complex non-Bragg scattering mechanism with high depolarization and high random (Minchew et al., 2012). At this moment, $\lambda_{1}$ is only slightly larger than $\lambda_{2}$ and $\lambda_{3}$, the value of the $r r r_{s}$ is low. The distributions of $r r r_{s}$ in water region, look-alikes region, and oil slicks region are shown in Fig.1. There are eleven black points in each column, corresponding to the $r r r_{s}$ value in $0 \%, 10 \%$, $20 \%, \ldots, 100 \%$ of each region samples, respectively. It is obvious that $r r r_{s}$ value distributions of oil slicks are different from lookalikes and water. Therefore, $r r r_{s}$ can be used to distinguish oil slicks from look-alikes.

\section{EXPERIMENT}

\subsection{Experiment Data}

In this paper, two different sensors data are used to verify the proposed method. The first is a RADARSAT2 image. It is a manmade experiment of oil spill by Norwegian Clean Seas Association for Operating Companies (NOFO). There are three different parts in this image, include plant oil, emulsion and crude oil. The experiment released $0.4 \mathrm{~m}^{3}$ Radiagreen ebo plant oil, the plant oil has the similar ambiphilic structure as the surface-active compounds in natural slicks. Therefore, the plant oil is used to simulate the natural monomolecular biogenic slicks. The experiment released about $1 \mathrm{~m}^{3}$ of emulsion on the surface, which consist of Oseberg blend crude oil and IFO380. The crude oil was consisted $30 \mathrm{~m}^{3}$ of evaporated Balder crude oil. These slicks were sprinkled onto sea surface $13 \mathrm{~h}, 29 \mathrm{~h}$, and $9 \mathrm{~h}$ before satellite imaging respectively (Skrunes et al., 2014). The Pauli RGB image of Radarsat-2 is shown in Fig. 2(a). The red box represents crude oil region, the yellow box represents Emulsion region, the green box represents plant oil region, and the blue box represents water region.

The second are two SIR-C images, their number are Pr17041 and Pr44327, respectively. It is a man-made experiment of oil spill by Jet Propulsion Laboratory (JPL), National Aeronautics and Space Administration (NASA) (Migliaccio et al., 2007). The two SIR$\mathrm{C}$ images only contain one kind of oil slicks. The Pauli RGB image of SIR-C are shown in Fig. 2(b) and 2(c). The red box represents oil region and the blue box represents water region. The details of the three experiment images are shown in Table 1.

\begin{tabular}{|c|c|c|c|}
\hline Data & Radarsat-2 & Pr17041 & Pr44329 \\
\hline $\begin{array}{c}\text { Area of } \\
\text { acquisition }\end{array}$ & North Sea & North Sea & North Sea \\
\hline $\begin{array}{c}\text { Data, time } \\
\text { UTC }\end{array}$ & $\begin{array}{c}\text { June 8, 2011 } \\
17: 27\end{array}$ & $\begin{array}{c}\text { October 1, } \\
199408: 14\end{array}$ & $\begin{array}{c}\text { April 11, } \\
199410: 49\end{array}$ \\
\hline Surfactant & $\begin{array}{c}\text { Oil and } \\
\text { look-alikes }\end{array}$ & Oil & Oil \\
\hline $\begin{array}{c}\text { Wind speed } \\
\text { (m/s) }\end{array}$ & $1.6 \sim 3.3$ & $\begin{array}{c}\text { Low to } \\
\text { moderate }\end{array}$ & $\begin{array}{c}\text { Low to } \\
\text { moderate }\end{array}$ \\
\hline $\begin{array}{c}\text { Incidence } \\
\text { angle }\left({ }^{\circ}\right)\end{array}$ & $34.48 \sim 36.08$ & $44.09 \sim 47.52$ & $35.48 \sim 40.45$ \\
\hline $\begin{array}{c}\text { Resolution } \\
(\mathrm{m} \times \mathrm{m})\end{array}$ & $5.2 \times 7.6$ & $25 \times 25$ & $25 \times 25$ \\
\hline
\end{tabular}

Table 1. The details of the three experiment images. 


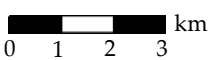

Central Latitude: $60^{\circ} 00^{\prime} 11^{\prime \prime} \mathrm{N}$ Central Longitude: $2^{\circ} 05^{\prime} 32^{\prime \prime} \mathrm{E}$

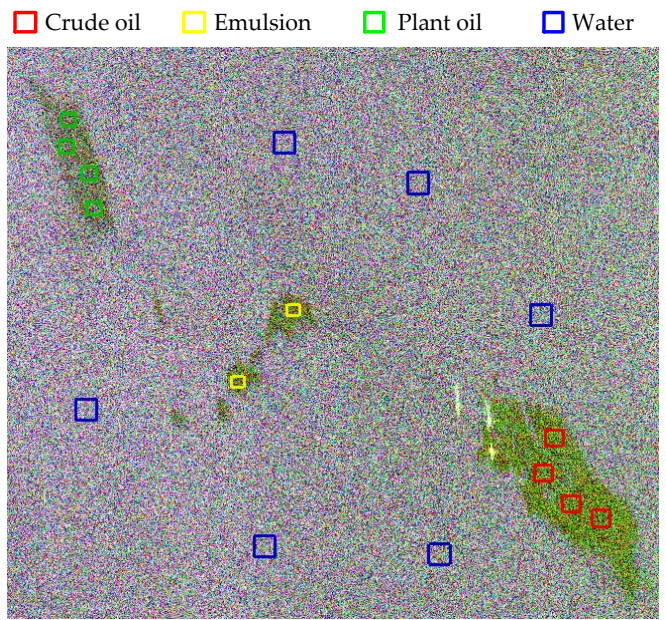

(a)

Central Latitude: $57^{\circ} 12^{\prime} 58^{\prime \prime} \mathrm{N}$
Central Longitude: $1^{\circ} 5^{\prime} 03^{\prime \prime} \mathrm{E}$ $\square$ Water

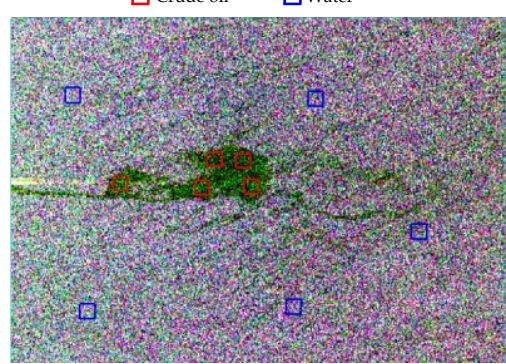

(b)

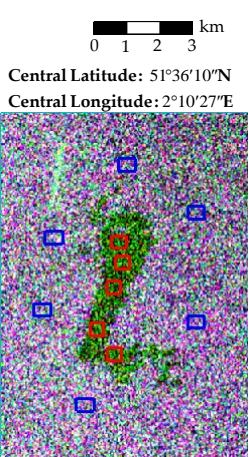

(c)

Figure 2. The PauliRGB images of experiment data. (a) Radarsat-2 image; (b) SIR-C Pr17041 image; (c) SIR-C Pr44327 image.

\subsection{Oil Spill Detection Capability Analysis of Polarimetric Features}

In order to prove that the self-similarity parameter can detect oil spill effectively, we compared $r r r_{s}$ with five commonly-used well-known polarimetric features in oil spill detection. They are degree of polarimetric (Dop) (Nunziata et al., 2013), conformity coefficient $(\mu)$ (Zhang et al., 2011), anti-entropy $\left(A_{12}\right)$ (Wang et al., 2010), pedestal height $(P h)$ (Nunziata et al., 2011), geometric intensity $(V)$ (Skrunes et al., 2014), respectively. These features are shown better ability of oil spill detection in the corresponding literature. The expressions for these features, along with their expected behaviour over sea surface with and without oil slicks, are described in Table 2.

\begin{tabular}{|c|c|c|c|}
\hline Feature & Expression & $\begin{array}{c}\text { Oil } \\
\text { slicks }\end{array}$ & $\begin{array}{c}\text { Non-oil } \\
\text { slicks }\end{array}$ \\
\hline Dop & Dop $=\sqrt{\frac{1}{3}\left(\frac{t r\left(M^{H} M\right)}{M_{l I}^{2}}-1\right)}$ & Low & High \\
\hline$\mu$ & $\mu=\frac{2\left(\operatorname{Re}\left\langle S_{H H} S_{V V}^{*}\right\rangle-\left|S_{H V}^{2}\right|\right)}{S p a n}$ & Low & High \\
\hline$A_{12}$ & $A_{12}=\frac{\lambda_{1}-\lambda_{2}}{\lambda_{1}+\lambda_{2}}$ & Low & High \\
\hline
\end{tabular}

\begin{tabular}{|c|c|c|c|}
\hline$P h$ & $P h=\frac{\lambda_{3}}{\lambda_{1}}$ & High & Low \\
\hline$V$ & $V=\operatorname{det}(T)^{3}$ & Low & High \\
\hline
\end{tabular}

Table 2. Polarimetric features and their expected behavior over sea surface with and without oil slicks.

According to the samples in Fig. 2(a), the Probability Density Functions (PDF) of six features in Radarsat-2 image are shown in Figure 3, which introduces the value distribution of different features in different regions. The more overlap area between two regions means that the weak discriminability of two regions, the less overlap area means the strong discriminability.

It can be seen in Figure 3 that in all the above features, the overlap area between the oil slicks and the water is relatively small, expect for a few overlap areas in Fig 3(a) and Fig 3(b), they are completely separate in other features, which means that the oil slicks and the water have a good distinction in these polarimetric features. The overlap area between two oil slicks (crude oil and emulsion) is relatively large in each feature, they even overlap directly in Fig 3(e). It means that the two oil slicks are hard to separate by these features. There is also the large overlap between the oil slicks and the plant oil, the size of overlap area is different in the different polarimetric features. In general, the overlap area of emulsion and plant oil is larger than the overlap area of crude oil and plant oil. From PDFs, it is obvious that there is less overlap areas between the oil slicks and the look-alikes in Fig 3(c), (d), (e), (f), and more overlap areas in Fig 3(a), (b), which means that the oil spill detection capability of $P h, V, A_{12}$, and $r r r_{s}$ is stronger than $D o P$, and $\mu$.

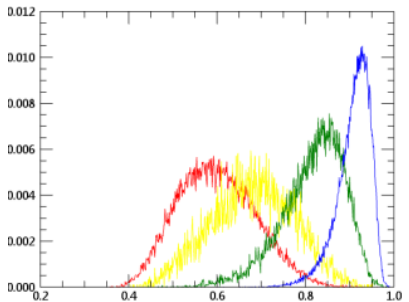

(a)

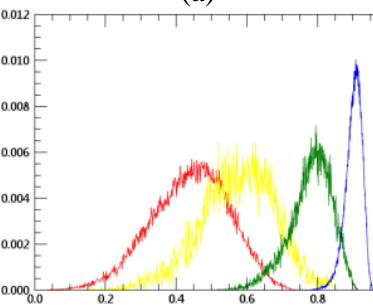

(c)

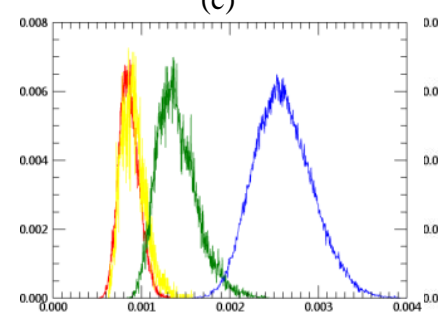

(e)

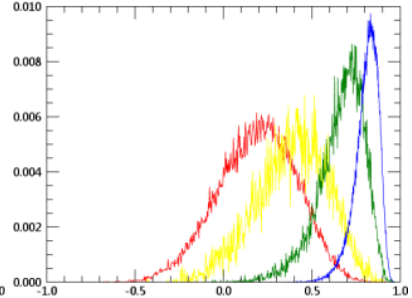

(b)

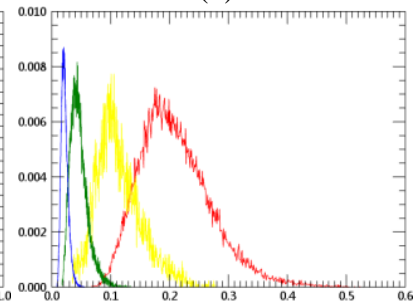

(d)

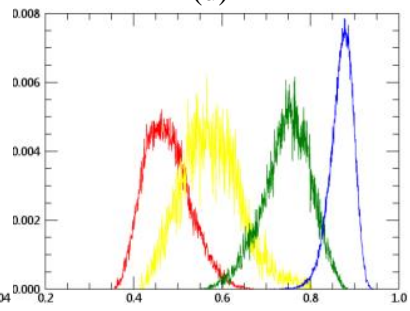

(f)
Figure 3. Probability Density Functions of features. (a) Dop; (b) $\mu$; (c) $A_{12}$; (d) $P h$; (e) $V$; (f) $r r r_{s}$.

In order to quantitative compare the detection capability of different polarimetric features in water, look-alikes, and oil slicks, 
the Normalized Distance (ND) was utilized to quantify the oil spill detection capability of polarimetric features (Li et al., 2015).

$$
d=\frac{\left|\mu_{1}-\mu_{2}\right|}{\delta_{1}+\delta_{2}}
$$

where, $\mu_{i}$ denotes the mean of sample, $\delta_{i}$ denotes the standard deviation of sample. Large ND between two regions means that these two regions are hard to distinguish. Otherwise, small ND means that these two regions are hard to distinguish.

\begin{tabular}{|c|c|c|c|}
\hline Feature & Radarsat-2 & SIR-C Pr17041 & SIR-C Pr44327 \\
\hline Dop & 2.0699 & 3.4925 & 2.7496 \\
\hline$\mu$ & 1.8684 & 3.3416 & 2.6650 \\
\hline$A_{12}$ & 3.0001 & 4.4009 & 4.1080 \\
\hline$P h$ & 2.2370 & 3.8189 & 3.1377 \\
\hline$V$ & 3.5258 & 3.1896 & 3.0254 \\
\hline$r r r_{s}$ & $\mathbf{3 . 8 7 6 7}$ & $\mathbf{6 . 3 8 6 7}$ & $\mathbf{5 . 2 3 6 3}$ \\
\hline
\end{tabular}

Table 3. The NDs between oil slicks and water of features in three SAR images.

\begin{tabular}{|c|c|c|c|c|}
\hline Feature & E vs P & C vs P & E vs W & C vs W \\
\hline Dop & 0.8674 & 1.2786 & 1.7296 & 2.2509 \\
\hline$\mu$ & 0.8766 & 1.2836 & 1.5496 & 1.9998 \\
\hline$A_{12}$ & 1.3943 & 1.9403 & 2.6301 & 3.2561 \\
\hline$P h$ & 1.3004 & 2.0378 & 2.0946 & 2.6722 \\
\hline$V$ & 1.4880 & 1.5104 & 3.0894 & 3.0944 \\
\hline$r r r_{s}$ & $\mathbf{1 . 5 4 8 9}$ & $\mathbf{2 . 4 6 4 6}$ & $\mathbf{3 . 1 8 8 0}$ & $\mathbf{4 . 5 3 8 0}$ \\
\hline
\end{tabular}

Table 4 . The NDs between oil slicks and non-slicks of features in Radarsat-2 image. Where C, E, P, and W refer to crude oil, emulsion, plant oil, and water respectively.

The NDs between oil slicks and water of six features in three images are shown in Table 3. Among them, crude oil and emulsion are counted as oil slicks in Radarsat- 2 image. It indicates the ability of these features to distinguish oil slicks from water. The NDs between different oil slicks and non-oil slicks of six features in Radarsat-2 image are shown in Table 4. It indicates the ability of these features to distinguish oil slicks (crude oil and emulsion) from non-oil slicks (look-alikes and water). Overall, Among the six features, each ND values of $r r r_{s}$ are the largest, it means that $r r r_{s}$ is better at detecting oil slicks than other features.

\subsection{Oil Spill Detection by Polarimetric Features}

These polarimetric features were classified using K-means classification for oil spill detection. Before detection, SAR images were processed with the Lee filter of a $7 \times 7$ window for speckle reduction. The accuracy of oil spill detection is evaluation by $F_{l}$ Score, which is a measure of accuracy of the classification result (Powers, 2011).

$$
F_{1}=\frac{2 *(1-F A R) * D R}{(1-F A R+D R)}
$$

where $D R$ defined the detection rate of oil slicks, FAR defined the false alarm rate of oil slicks.

The K-means classifications result of polarimetric features in three images are shown in Fig 4, Fig 5, and Fig 6, corresponding Radarsat-2, Pr17041, and Pr44329 image respectively. The $F_{l}$ scores of these classification results are shown in Table 5.

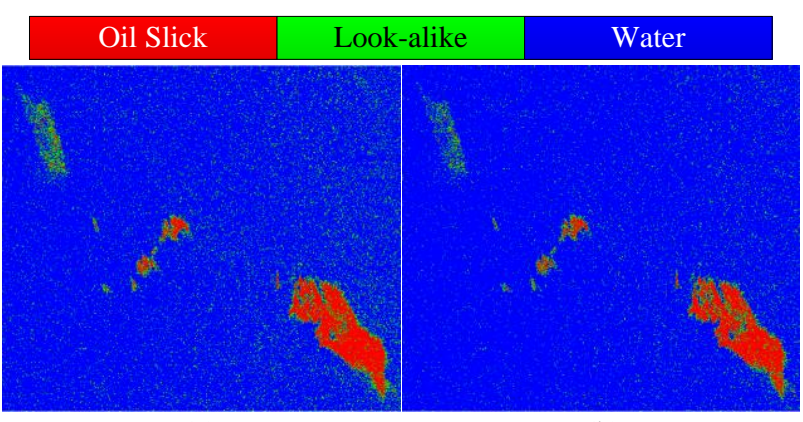

(a)

(b)

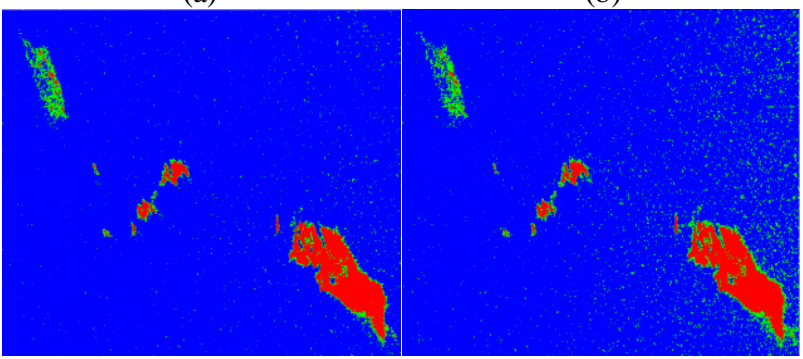

(c)

(d)

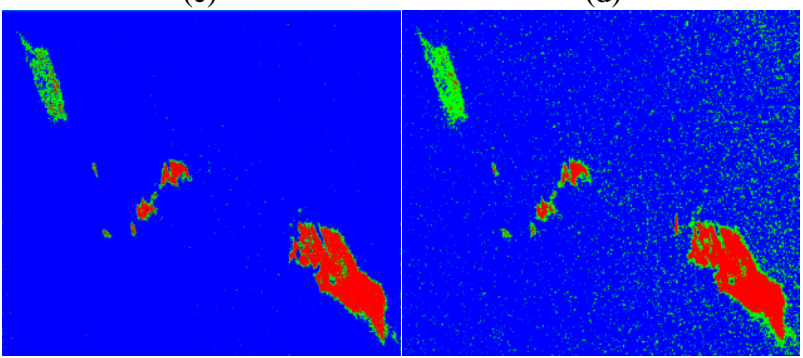

(e)

(f)

Figure 4. K-means classification of six polarimetric features in Radarsat-2 images. (a) $D o p$; (b) $\mu$; (c) $A_{12}$; (d) $P h$; (e) $V$; (f) $r r r_{s}$.

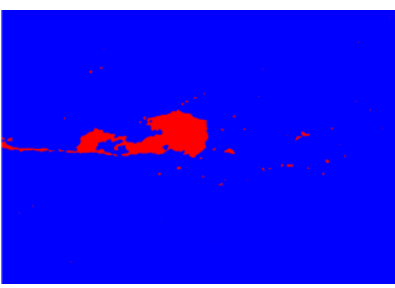

(a)

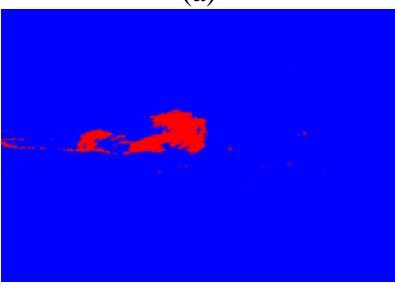

(c)

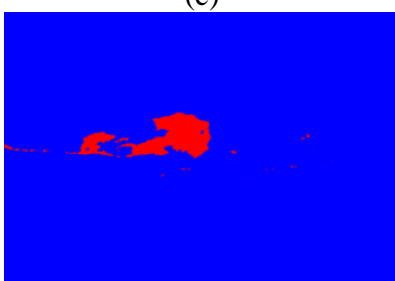

(e)

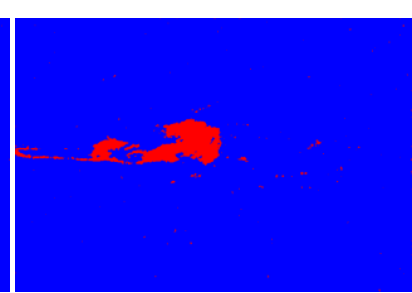

(b)

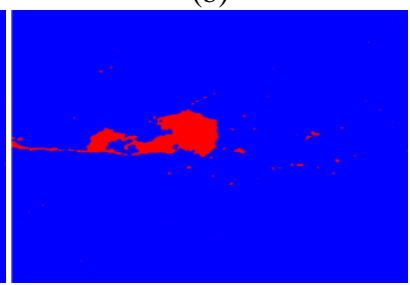

(d)

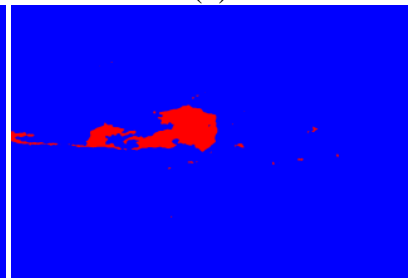

(f)
Figure 5. K-means classification of six polarimetric features in SIR-C Pr17041 images. (a) Dop; (b) $\mu$; (c) $A_{12}$; (d) $P h$; (e) $V$; (f) $\mathrm{rrr}_{\mathrm{s}}$. 


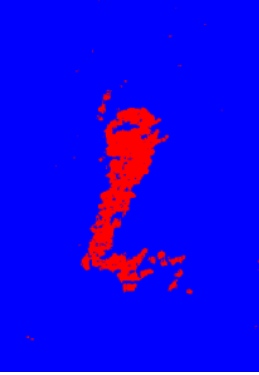

(a)

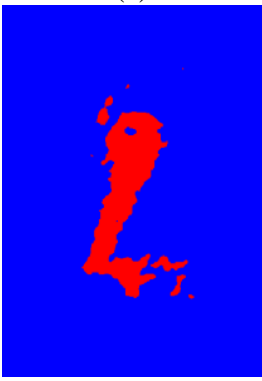

(d)

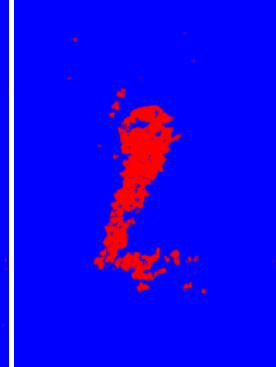

(b)

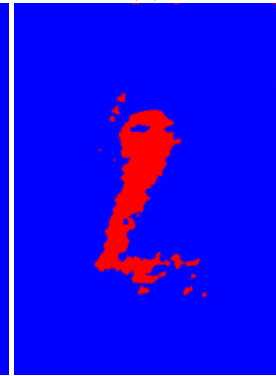

(e)

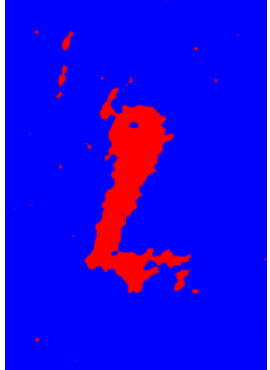

(c)

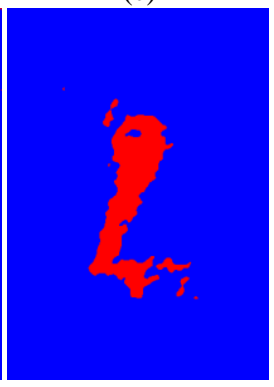

(f)
Figure 6. K-means classification of six polarimetric features in SIR-C Pr44327 images. (a) Dop; (b) $\mu$; (c) $A_{12}$; (d) $P h$; (e) $V$; (f) $\operatorname{rrr}_{s}$.

\begin{tabular}{|c|c|c|c|}
\hline & Radarsat-2 & SIR-C Pr17041 & SIR-C Pr44327 \\
\hline Dop & $80.06 \%$ & $86.48 \%$ & $82.86 \%$ \\
\hline$\mu$ & $79.18 \%$ & $86.43 \%$ & $83.13 \%$ \\
\hline$A_{12}$ & $90.55 \%$ & $89.85 \%$ & $89.36 \%$ \\
\hline$P h$ & $87.80 \%$ & $88.58 \%$ & $89.61 \%$ \\
\hline$V$ & $87.44 \%$ & $87.71 \%$ & $88.68 \%$ \\
\hline$r r r_{s}$ & $\mathbf{9 1 . 5 7 \%}$ & $\mathbf{9 1 . 0 4 \%}$ & $\mathbf{9 0 . 4 8 \%}$ \\
\hline
\end{tabular}

Table 5 . The $F_{1}$ score of classification using different features within three images.

In Fig. 4, it is obvious that in plant oil region, misclassified as oil slicks of $\operatorname{rrr}_{s}$ is less than other features. In addition, at the interface between the oil slicks (include crude oil and emulsion) and the water, some pixels are classified as plant oil. These misclassified pixels are mainly water, they formed a thinner film at the edge of the oil slicks, and the scattering mechanism of this film is closer to the plant oil. Overall, $r r r_{s}$ distinguishes the oil slicks from look-alikes better than other polarimetric features. In Fig. 5 and Fig. 6, oil slicks and water have the better distinction in each feature, but the oil spill detection in $r r r_{s}$ is still more complete than other features. The $F_{l}$ score shown in Table 5 also proves $\mathrm{rrr}_{s}$ is more effective than other polarimetric features in oil spill detection.

\section{CONCLUSION}

In this paper, the self-similarity parameter, which is sensitive to the randomness of the target scattering mechanism, is introduced to reflect the scattering mechanism of the oil slicks and to distinguish the oil slicks from the look-alikes. According to the analysis results of PDFs and NDs, the self-similarity parameter of oil spill detection ability is superior to other well-known polarimetric features. In addition, the $F_{1}$ score of oil spill detection using self-similarity parameter is higher than other features. Therefore, it is proved that the self-similarity parameters can detect oil slicks effectively.

\section{ACKNOWLEDGEMENTS}

This work is supported in part by the National Natural Science Foundation of China under Grant No. 41771467 and No. 41471355.

\section{REFERENCES}

Brekke, C., Solberg, A.H.S., 2005. Oil Spill Detection by Satellite Remote Sensing. Remote Sens. Environ. 95(1), pp. 1-13.

Chen, Q., Jiang, Y.M., Zhao, L.J., Kuang, G.Y., 2010. Polarimetric Scattering Similarity Between a Random Scatterer and a Canonical Scatterer. IEEE Geosci. Remote Sens. 7(4), pp. 866-869.

Li, D., Zhang, Y., 2015. Random Similarity Between Two Mixed Scatterers. IEEE Geosci. Remote Sens. 12(12), pp. 2468-2472.

Li, X., Zheng, W., Yang, X., Pietrafesa, L.J., 2017. Sea Fetch Observed by Synthetic Aperture Radar. IEEE Trans. Geosci. Remote Sens. 55(1), pp. 272-279.

Li, Y., Lin, H., Zhang, Y., Chen, J., 2015. Comparisons of Circular Transmit and Linear Receive Compact Polarimetric SAR Features for Oil Slicks Discrimination. J. Sensors. 2015(99), $1-14$.

Liu, P., Li, X., Qu, J.J., Wang, W., Zhao, C., Pichel, W., 2011. Oil Spill Detection with Fully Polarimetric UAVSAR Data. Mar Pollut Bull. 62(12), pp. 2611-8.

Migliaccio, M., Gambardella, A., Tranfaglia, M., 2007. SAR Polarimetry to Observe Oil Spills. IEEE Trans. Geosci. Remote Sens. 45(2), pp. 506-511.

Migliaccio, M., Nunziata, F., Buono, A., 2015. SAR Polarimetry for Sea Oil Slicks Observation. Int J. Remote Sens. 36(12), pp. 3243-3273.

Migliaccio, M., Nunziata, F., Gambardella, A., 2007. On the Copolarised Phase Difference for Oil Spill Observation. Int J. Remote Sens. 45(2), pp. 506-511.

Migliaccio, M., Nunziata, F., Montuori, A., Li, X., Pichel, W.G., 2012. A Multifrequency Polarimetric SAR Processing Chain to Observe Oil Fields in the Gulf of Mexico. IEEE Geosci. Remote Sens. 49(12), pp. 4729-4737.

Migliaccio, M., Tranfaglia, M., Ermakov, S.A., 2005. A Physical Approach for the Observation of Oil Spills in SAR Images. IEEE J. Oceanic Eng. 30(3), 496-507.

Minchew, B., Jones, C.E., Holt, B., 2012. Polarimetric Analysis of Backscatter from the Deepwater Horizon Oil Spill Using LBand Synthetic Aperture Radar. IEEE Trans. Geosci. Remote Sens. 50(10), 3812-3830.

Nunziata, F., Gambardella, A., Migliaccio, M., 2013. On the Degree of Polarization for SAR Sea Oil Slicks Observation. Isprs J Photogramm. 78(4), pp. 41-49.

Nunziata, F., Migliaccio, M., Gambardella, A., 2011. Pedestal Height for Sea Oil Slicks Observation. Iet Radar Sonar Nav. 5(2), pp. 103-110.

Powers, D.M.W., 2011. Evaluation: From Precision, Recall and 
F-Factor to ROC, Informedness, Markedness and Correlation. Journal of Machine Learning Technologies. 2(1), pp. 2229-3981.

Skrunes, S., Brekke, C., Eltoft, T., 2014. Characterization of Marine Surface Slicks by Radarsat-2 Multipolarization Features. IEEE Trans. Geosci. Remote Sens. 52(9), pp. 5302-5319.

Van Zyl, J.J., 1989. Unsupervised Classification of Scattering Behavior Using Radar Polarimetry Data. IEEE Trans. Geosci. Remote Sens. 27(1), pp. 36-45.

Wang, W., Lu, F., Wu, P., Wang, J., 2010. Oil Spill Detection from Polarimetric SAR Image. IEEE, International Conference on Signal Processing. pp. 832-835.

Yang, J., Dong, G., Peng, Y., Yamaguchi, Y., 2004. Generalized Optimization of Polarimetric Contrast Enhancement. IEEE Geosci. Remote Sens. 1(3), pp. 171-174.

Yang, J., Peng, Y.N., Lin, S.M., 2001. Similarity Between Two Scattering Matrices. Electron Lett. 37(3), pp 193-194.

Zhang, B., Perrie, W., Li, X., Pichel, W.G., 2011. Mapping Sea Surface Oil Slicks Using RADARSAT-2 Quad-Polarization SAR Image. Geophys Res Lett. 38(10), pp. 415-421. 